\title{
Bogoliubov Inequalities for Infinite Systems *
}

\author{
J. C. GARrison and J. WonG \\ Lawrence Radiation Laboratory, University of California, Livermore, California USA
}

Received August 9, 1971

\begin{abstract}
The Bogoliubov inequalities are derived for the infinite volume states describing the thermodynamic limits of physical systems. The only property of the states required is that they satisfy the Kubo-Martin-Schwinger boundary condition.
\end{abstract}

\section{Introduction}

In the conventional Green's function approach to statistical mechanics all relations are first derived for strictly finite systems; the thermodynamic limit is taken at the end of the calculation. Since the original derivation [1] of the Bogoliubov inequalities was carried out within this framework, the subsequent applications had to follow the same prescription. In this way the inequalities have been applied by Josephson [2] to derive rigorous inequalities for the specific heat and by a number of authors [3-7] to show the impossibility of various kinds of long-range order in one- and two-dimensional systems. In the latter class of problems, a special difficulty arises from the fact that finite systems do not exhibit the broken symmetries usually associated with long-range order. This has led to the use of Bogoliubov's quasi-averaging method in which the finite-system Hamiltonian is modified by the addition of a symmetry breaking term, which is set equal to zero only after the passage to the thermodynamic limit. This method has never been shown to be equivalent to the more rigorous treatment of broken symmetries provided by the theory of integral decompositions of states on $C^{*}$-algebras $[8,9]$; furthermore, for some problems (e.g. Bose condensation and anti-ferromagnetism) the symmetry breaking term has no physical significance.

The purpose of this paper is to show how these difficulties can be avoided by establishing the Bogoliubov inequalities directly in the thermodynamic limit. An application of the inequalities to long-range order in one- and two-dimensional systems will be published separately.

* This work was performed under the auspices of the U.S. Atomic Energy Commission.

1 Commun math. Phys., Vol. 26 


\section{Theoretical Framework and Physical Assumptions}

The appropriate setting for our derivation is provided by the algebraic approach to statistical mechanics; in particular we will make extensive use of the results of Haag, Hugenholtz, and Winnink [10]. Let $\omega$ denote a state on the algebra $\mathfrak{A}$ of quasi-local observables. A standard construction associates with $\omega$ and $\mathfrak{Q}$ a Hilbert space $\mathfrak{H}$ and a representation of $\mathfrak{U}$ by bounded operators on $\mathfrak{H}$. Physical symmetry operations are represented by groups of automorphisms on $\mathfrak{A}$ and in some cases by unitary transformations on $\mathfrak{S}$.

We restrict our attention to states which satisfy the Kubo-MartinSchwinger (KMS) boundary condition. It is known that KMS states are invariant under time translations, which implies that time translations are represented by unitary operators on $\mathfrak{H}$. The precise statement of the KMS condition is

$$
\int d t f(t-i \beta) \omega\left(B A_{t}\right)=\int d t f(t) \omega\left(A_{t} B\right),
$$

where $A \rightarrow A_{t}$ is the time translation automorphism. This equation is to hold for all $f \in \mathscr{D}$ (the space of $C^{\infty}$ functions with compact support) and all $A, B \in \mathfrak{H}$.

In their analysis of the KMS condition Haag, Hugenholtz, and Winnink introduced a subalgebra $\tilde{\mathfrak{A}} \subset \mathfrak{A}$ as follows: For each $A \in \mathfrak{A}$ define the operator-valued distribution

$$
\hat{A}(\varepsilon)=\int d t e^{i \varepsilon t} A_{t},
$$

which exists by virtue of the fact that $A_{t}$ is a bounded (operator) function of $t$. Then $\tilde{\mathfrak{Q}}$ is defined to be the algebra generated by all $A$ for which $\hat{A}$ has compact support. We list here several properties of these operators which will be useful later on. In the first place, the Fourier transform of a distribution with compact support is an entire function for complex values of its argument [12]. Thus, on $\tilde{\mathfrak{A}}$ the real time-translations can be extended to complex time, $\eta$, by the automorphism

$$
A \rightarrow A_{\eta}=\int d \varepsilon e^{i \eta \varepsilon} \hat{A}(\varepsilon),
$$

which satisfies $\left(A^{\dagger}\right)_{\eta}=\left(A_{\eta^{*}}\right)^{\dagger}$ and $\omega\left(A_{\eta}\right)=\omega(A)$. The latter condition is a consequence of the invariance of $\omega$ under real time-translations. For $A$ and $B \in \tilde{\mathfrak{A}}$ we can replace (1) by

$$
\omega\left(B A_{t+i \beta}\right)=\omega\left(A_{t} B\right) .
$$

It is also true that a distribution with compact support is a continuous linear functional on the space, $\mathscr{E}$, of $C^{\infty}$ functions without any growth restrictions at infinity [13]. 
Finally, we remark that $\tilde{\mathfrak{A}}$ is norm-dense in $\mathfrak{A}$. To see this, note that if $A \in \mathfrak{A}$ and $\hat{f} \in \mathscr{D}$, we have

$$
A_{f} \equiv \int d t f(t) A_{t} \in \tilde{\mathfrak{I}} .
$$

Let $\sigma$ be a norm-continuous linear functional on $\mathfrak{A}$, and suppose that $\sigma$ vanishes on each $A_{y}$, then

$$
0=\int d t f(t) \sigma\left(A_{t}\right)
$$

for all $A \in \mathfrak{A}$ and $\hat{f} \in \mathscr{D}$. The functions $\not$ having $\hat{f} \in \mathscr{D}$ are dense in $\mathscr{S}$ (the space of $C^{\infty}$ functions vanishing rapidly at infinity), and the bounded function $\sigma\left(A_{t}\right)$ determines an element of the dual space $\mathscr{S}^{\prime}$; therefore, the above condition can be extended to all $f \in \mathscr{S}$. It follows that $\sigma\left(A_{t}\right) \equiv 0$; in particular, $\sigma(A)=0$ for all $A \in \mathfrak{A}$. Consequently, any continuous linear functional which annihilates $\tilde{\mathfrak{A}}$ necessarily vanishes; this implies that $\tilde{\mathfrak{U}}$ is dense in $\mathfrak{A}$.

\section{The Bogoliubov Inequalities}

For $A, B \in \tilde{\mathfrak{A}}$ we assert that the following equivalent expressions define an inner product:

$$
\begin{aligned}
(A, B) & =\frac{1}{\beta} \int_{0}^{\beta} d \tau \omega\left(\left(A^{\dagger}\right)_{-i \tau} B\right), \\
& =\frac{1}{\beta} \int_{0}^{\beta} d \tau \omega\left(\left(A_{i \tau}\right)^{\dagger} B\right) .
\end{aligned}
$$

Since $\omega$ is a state, these expressions are evidently linear in $B$, but the other necessary features of an inner product depend on the property of time translation invariance. We have

and

$$
(A, B)^{*}=\frac{1}{\beta} \int_{0}^{\beta} d \tau \omega\left(\left(B^{\dagger}\right)_{-i \tau} A\right)=(B, A)
$$

$$
\begin{aligned}
(A, A) & =\frac{1}{\beta} \int_{0}^{\beta} d \tau \omega\left(\left(A^{\dagger}\right)_{-\frac{i \tau}{2}} A_{\frac{i \tau}{2}}\right), \\
& =\frac{1}{\beta} \int_{0}^{\beta} d \tau \omega\left(\left(A_{\frac{i \tau}{2}}\right)^{\dagger} A_{\frac{i \tau}{2}}\right) \geqq 0 .
\end{aligned}
$$

Thus, the time translation invariance of $\omega$ is sufficient to ensure that $(A, B)$ is an inner product; in particular, one obtains the Schwarz inequality

$$
|(A, B)|^{2} \leqq(A, A)(B, B) .
$$

To derive the Bogoliubov inequality from (2), we need some special properties of this inner product which follow from the KMS boundary 
condition First, choose $B=i(\partial / \partial t) C_{t \mid t=0}$ and use time translation invariance to write

$$
(A, B)=\frac{1}{\beta} \int_{0}^{\beta} d \tau \omega\left(A^{\dagger} i \frac{\partial}{\partial t} C_{t+i \tau \mid t=0}\right) .
$$

In the integrand we can replace $i(\partial / \partial t)$ by $(\partial / \partial \tau)$ and evaluate the integral to get

$$
\begin{aligned}
(A, B) & =\frac{1}{\beta}\left[\omega\left(A^{\dagger} C_{i \beta}\right)-\omega\left(A^{\dagger} C\right)\right] \\
& =\frac{1}{\beta} \omega\left(\left[C, A^{\dagger}\right]\right),
\end{aligned}
$$

where the last line follows from the KMS condition. Next, we obtain an upper bound on $(A, A)$ as follows:

$$
\begin{aligned}
(A, A) & =\frac{1}{\beta} \int_{0}^{\beta} d \tau \omega\left(A^{\dagger} A_{i \tau}\right) \\
& =\frac{1}{\beta} \int_{0}^{\beta} d \tau \int d \varepsilon e^{\tau \varepsilon} \omega\left(A^{\dagger} \hat{A}(\varepsilon)\right), \\
& =\int d \varepsilon \frac{e^{\beta \varepsilon}-1}{\beta \varepsilon} \omega\left(A^{\dagger} \hat{A}(\varepsilon)\right) .
\end{aligned}
$$

The last step is justified because the first factor is a $C^{\infty}$ function and the second is a distribution with compact support. Furthermore, $\omega\left(A^{\dagger} A_{t}\right)$ is a positive definite function, which means that $\omega\left(A^{\dagger} \hat{A}(\varepsilon)\right)$ is a positive distribution. This fact, together with the elementary inequality

leads to

$$
\frac{e^{x}-1}{x} \leqq \frac{e^{x}+1}{2}
$$

$$
\begin{aligned}
(A, A) & \leqq \int d \varepsilon \frac{e^{\beta \varepsilon}+1}{2} \omega\left(A^{\dagger} \hat{A}(\varepsilon)\right), \\
& =\frac{1}{2}\left[\omega\left(A^{\dagger} A_{i \beta}\right)+\omega\left(A^{\dagger} A\right)\right], \\
& =\omega\left(\frac{1}{2}\left\{A, A^{\dagger}\right\}\right) .
\end{aligned}
$$

These results have been derived for elements of $\tilde{\mathfrak{A}}$; however, we have already shown, in Section II, that $\tilde{\mathfrak{A}}$ is dense in $\mathfrak{A}$, and we will now show that the inner product is continuous. Continuity follows from the estimates

$$
\begin{gathered}
|(A, B)| \leqq[(A, A)(B, B)]^{1 / 2}, \\
(A, A) \leqq \omega\left(\frac{1}{2}\left\{A, A^{\dagger}\right\}\right) \leqq\|A\|^{2} ;
\end{gathered}
$$


thus, $|(A, B)| \leqq\|A\| \cdot\|B\|$. The inner product is then defined on all of $\mathfrak{A}$ by continuity.

Finally, we obtain the Bogoliubov inequalities by combining (2), (3), and (4).

$$
\omega\left(\left\{A, A^{\dagger}\right\}\right) \omega\left(\left[C,\left(i \frac{\partial C_{t}}{\partial t}\right)_{0}^{\dagger}\right]\right) \geqq \frac{2}{\beta}\left|\omega\left(\left[C, A^{\dagger}\right]\right)\right|^{2} .
$$

This relation assumes a more familiar form if we pass from the algebra $\mathfrak{A}$ to its representation on $\mathfrak{S}$. We then have a unitary group $U_{t}$ with a corresponding generator $H$ in terms of which we can write (with the same notation for elements of $\mathfrak{U}$ and their representative operators),

$$
i\left(\frac{\partial}{\partial t} C_{t}\right)_{0}=[C, H] \text {. }
$$

Using the conventional notation, $\omega(A)=\langle A\rangle$, (5) can be rewritten as

$$
\left\langle\left\{A, A^{\dagger}\right\}\right\rangle\left\langle\left[C,\left[H, C^{\dagger}\right]\right]\right\rangle \geqq \frac{2}{\beta}\left|\left\langle\left[C, A^{\dagger}\right]\right\rangle\right|^{2},
$$

which holds for all $A \in \mathfrak{A}$. The inequality also holds for physical quantities represented by unbounded operators provided only that their spectral resolutions lie in $\mathfrak{A}$. In such a case, we can obtain the unbounded operator as a limit of a sequence of elements of $\mathfrak{A}$ and if the limits of the various expectation values all exist then (6) will continue to hold.

\section{References}

1. Bogoliubov, N. N.: Lectures on quantum statistics, Vol. 2, cf. p. 41 ff. New York: Gordon and Breach 1970.

2. Josephson, B. D.: Proc. Phys. Soc. 92, 269 (1967).

3. Hohenberg, P. C.: Phys. Rev. 158, 383 (1966).

4. Mermin, N.D.: J. Math. Phys. 8, 1061 (1966).

5. - Phys. Rev. 176, 250 (1968).

6. Chester, G. V., Fisher, M.E., Mermin, N.D.: Phys. Rev. 185, 760 (1969).

7. Fernández, J.F.: Phys. Rev. A 3, 1104 (1971).

8. Ruelle, D.: Commun. math. Phys. 3, 133 (1966).

9. Kastler, D., Robinson, D. W.: Commun. math. Phys. 3, 151 (1966).

10. Haag, R., Hugenholtz, N. M., Winnink, M.: Commun. math. Phys. 5, 215 (1967).

11. Ruelle,D.: Cargèse lectures in physics, Vol. 4, p. 169 ff. New York: Gordon and Breach 1970.

12. Schwartz, L.: Théorie des distributions, cf. p. 272. Paris: Hermann 1966.

13. - Théorie des distributions, p. 87 ff. Paris: Hermann 1966.

J. C. Garrison

J. Wong

Lawrence Livermore Lab.

Livermore, Cal. 94550, USA 